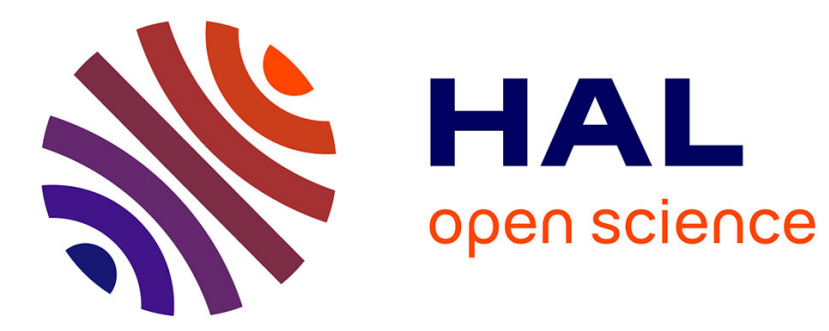

\title{
Observations of a streak texture in the hybrid-aligned smectic-C phase
}

I R Nemitz, I. Gryn, N. Boudet, R. P Lemieux, M. Goldmann, B. Zappone, R. G Petschek, C. Rosenblatt, Emmanuelle Lacaze

\section{To cite this version:}

I R Nemitz, I. Gryn, N. Boudet, R. P Lemieux, M. Goldmann, et al.. Observations of a streak texture in the hybrid-aligned smectic-C phase. Soft Matter, 2018, 14 (3), pp.460 - 469. 10.1039/C7SM02129A . hal-01688472

\section{HAL Id: hal-01688472 https://hal.sorbonne-universite.fr/hal-01688472}

Submitted on 19 Jan 2018

HAL is a multi-disciplinary open access archive for the deposit and dissemination of scientific research documents, whether they are published or not. The documents may come from teaching and research institutions in France or abroad, or from public or private research centers.
L'archive ouverte pluridisciplinaire $\mathbf{H A L}$, est destinée au dépôt et à la diffusion de documents scientifiques de niveau recherche, publiés ou non, émanant des établissements d'enseignement et de recherche français ou étrangers, des laboratoires publics ou privés. 


\title{
Observations of a streak texture in the hybrid-aligned Smectic-C phase
}

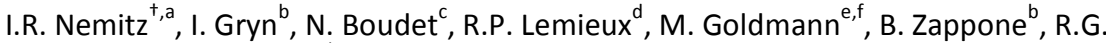 \\ Petschek $^{a}$, C. Rosenblatt ${ }^{*}, \mathrm{a}$, and E. Lacaze ${ }^{\mathrm{a}, \mathrm{e}}$
}

A novel structure was observed below the Smectic- $A$ - Smectic- $C$ phase transition in a very thin open cell having an air interface above and enforced planar anchoring at the substrate below. The structure appears as periodic dark and light streaks running perpendicular to the oily streaks, which are present in the Smectic-A phase [Soft Matter 12, 678 (2016)]. These new streaks, which we call "soapy streaks", form by extending from one oily streak to the next in discrete steps, eliminating optical evidence at visible wavelengths of the oily streaks. At lower temperatures the streaks can undulate and exhibit a sawtooth-like structure; such a structure is chiral in two dimensions. A possible scenario for the origin of these streaks is presented.

\section{Introduction}

Periodic microstructures often occur in Smectic-A (Sm-A) films when the film is subjected to competing boundary conditions at the two interfaces. These can be twodimensional when there is a $2 \pi$ azimuthal degeneracy of the boundary conditions ${ }^{2-6}$, or one dimensional if this azimuthal degeneracy is broken at a planar-orienting substrate ${ }^{7-12}$. For the latter case, i.e., the so-called Sm-A "oily streaks", in the picture to date smectic layers through most of the sample thickness remain parallel to the air interface (Fig. 1). At the substrate interface the smectic layers are normal to the substrate surface, with the director lying along the planar easy axis direction. In order to satisfy these competing boundary conditions, the smectic layers bend so as to form an array of hemicylinders with a central defect. The result is a series of periodic stripes, i.e., the oily streaks, along a direction perpendicular to the substrate's easy axis. Because the director at the liquid crystal / substrate interface presents planar alignment along a specified easy axis, when imaged by an optical microscope under crossed polarizers the entire sample appears dark if the director is oriented parallel to either of the polarizers. This oily streak texture has been investigated extensively, and has been observed in liquid crystals such as octylcyanobiphenyl (8CB) on $\mathrm{MoS}_{2}$ surfaces ${ }^{7,13-}$ ${ }^{15}$ and on cleaved mica ${ }^{12}$, on polyvinyl on alcohol (PVA) coated

a. Dept. of Physics, Case Western Reserve University, Cleveland, Ohio 44106 USA b. LiqCryl, University of Calabria, Rende, Italy

c. Univ. Grenoble Alpes, CNRS, Grenoble INP, Institut Néel, 38000 Grenoble, France.

d. Dept. of Chemistry, University of Waterloo, Waterloo, Ontario, Canada N2L $3 G 1$

e. Sorbonne Universités, UPMC Univ Paris 06, CNRS UMR 7588, Institut des NanoSciences de Paris (INSP), F75005 Paris, France

f. Synchrotron SOLEIL, L'Orme des Merisiers, Saint-Aubin, BP 48, 91192 Gif-surYvette, France

+ Current address: NASA Glenn Research Center, Brook Park, Ohio 44135 USA *Author for correspondence, rosenblatt@case.edu

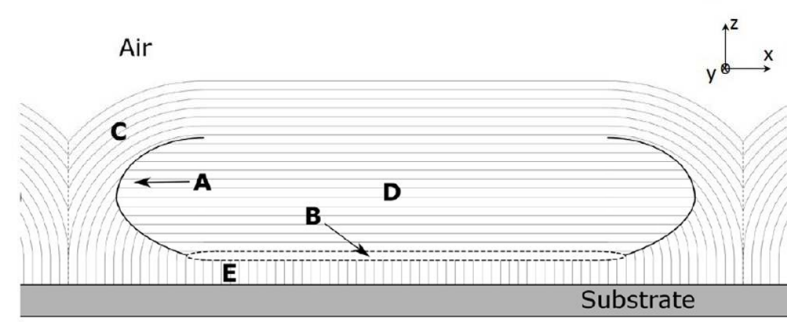

Fig. 1 Easy axis along $\hat{x}$. Structure of Sm-A oily streaks, where the thin lines represent smectic layers, A represents the rotating grain boundary (RGB), B a possibly melted nematic region, $\mathbf{C}$ the curvature walls, $\mathbf{D}$ the central homeotropic region, and $\mathbf{E}$ the planar transition region near the MoS, substrate.

surfaces $^{16,17}$, and also on polyimide coated surfaces ${ }^{18,19}$. They also have been observed between two substrates, polyimide coated surfaces, and a monolayer of silane surfactant, OTS, the latter inducing homeotropic alignment ${ }^{19}$.

Figure 1 presents a cartoon of the current picture, which is consistent with thin films $(\sim 120-300 \mathrm{~nm})$ of the liquid crystal $8 C B$ on a PVA alignment layer, recently observed using a combination of optical microscopy, ellipsometry, and x-rays ${ }^{16}$. On this substrate, it is known that the planar transition layer covers the entire alignment layer ${ }^{16}$. On PVA, it is most likely that the quasi-2D defect (region $B$ ) is a melted nematic phase rather than a smectic grain boundary ${ }^{16}$. In Fig. 1 the region marked $A$ is a rotating grain boundary (RGB), that also has been observed on $\mathrm{MoS}_{2}$ substrates ${ }^{7,13,15}$, B a possibly melted nematic region, $C$ the curvature walls, $D$ the central homeotropic region, and $\mathrm{E}$ the planar transition region near the substrate ${ }^{16}$. In this article we shall refer to the region $B$ as the central defect.

One reason underlying the extensive research conducted on oily streaks relates to the associated dense array of dislocations that allows for nanorod orientation ${ }^{20,21,22}$ and 
nanosphere/nanorod chain formation $22,23,24$. In this paper we examine the transformation of oily streaks from the Sm-A to the smectic-C $(\mathrm{Sm}-\mathrm{C})$ phase, but in the absence of nanoparticles. The effects of nanoparticles at this transition will be discussed in a future work.

In the lower symmetry Sm-C phase there are reports of structures that also consist of linear arrays. These have been shown to occur in freely suspended films ${ }^{25-28}$ as well as in systems with competing alignment ${ }^{29,30}$. In this latter arrangement, yet a new competition emerges: The director not only must match the air and substrate boundary conditions, but is no longer normal to the Sm-C layers. Here, we present empirical evidence that this competition in the Sm$C$ phase results in a novel structure, viz., as a series of straight streaks that appears in thin hybrid films of Sm-C liquid crystal. These streaks run perpendicular to oily streaks and grow in a discontinuous and incremental manner. As these new streaks grow, they replace optical evidence of the oily streaks; therefore we shall call them "soapy streaks"§. It is believed that as the temperature is reduced, the layered smectic structure of the system undergoes a rearrangement in the vicinity of the Sm-A to Sm-C phase transition. In the Sm-A oily streaks, where the layers curve toward the substrates ${ }^{12}$, an energy barrier must be overcome for the creation and propagation of the new structures. On cooling deeper into the $\mathrm{Sm}-\mathrm{C}$ phase, these straight soapy streaks start to undulate and become wavy, and in the thicker regions of the film appear to transform into an even more complicated series of sawtoothlike patterns. We report on the appearance and characteristics of these novel structures, and speculate on their makeup and the mechanisms behind their formation in thin layers of Sm-C liquid crystal. This will require some modifications of our picture of the oily streaks, but which will remain consistent with $\mathrm{x}$-ray and optical observations of the oily streaks.

\section{Experimental Methods}

\subsection{Sample Preparation}

The liquid crystal 9004 (Fig. 2 , with phase sequence in the

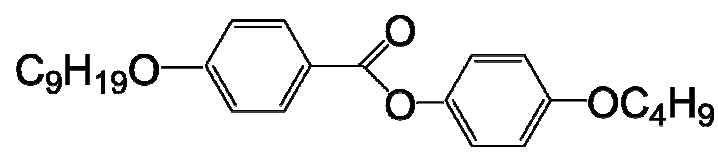

Fig. 2 Structure of liquid crystal 9004

bulk: Iso - 87 - Nematic - 73-Sm-A - 62-Sm-C - 50-Sm-B $35^{\circ} \mathrm{C}$ - Crystal), was used for most experiments presented herein. Soapy streaks were created following procedures similar to that used to form oily streaks in open cells: a hybridaligned liquid crystal film with planar alignment at the substrate, and a free surface (air) at the top with a homeotropic alignment ${ }^{16,18,19}$. Three different alignment layers were used to obtain the planar surface alignment: crystalline $\mathrm{MoS}_{2}$, rubbed PVA alignment layers, and rubbed polyimide alignment layers. The naturally occurring $\mathrm{MoS}_{2}$ crystals were supplied by The Ward Company (New York). Fresh substrates - only a few microns thick and $\sim 1 \mathrm{~cm}^{2}$ in area - were cleaved from the bulk crystals using adhesive tape (similar to the adhesive tape exfoliation method for obtaining graphene ${ }^{31}$ ). Due to the fragility of these $\mathrm{MoS}_{2}$ substrates, they were mounted on microscope slides with either epoxy or double-sided adhesive tape. Once stabilized, the fresh substrates were coated with 9004 above the liquid crystal's isotropic - nematic phase transition temperature, $\mathrm{T}_{\mathrm{NI}}$. This resulted in a thin film, typically between 70 and $350 \mathrm{~nm}$, over much of the $\mathrm{MoS}_{2}$. The sample then was allowed to cool to room temperature, which corresponds to the bulk crystalline phase of 9004.

In addition to $\mathrm{MoS}_{2}$, experiments were performed using rubbed polyimide alignment layers and polyvinyl alcohol (PVA). Here a reflective Si-protected aluminum substrate was spincoated with either the polyamic acid RN-1175 (Nissan Chemical Industries) or PVA. For certain experiments, glass slides also were coated. The surfaces were spin-coated at 2000 $\mathrm{rpm}$ for $20 \mathrm{~s}$ with the polyamic acid RN-1175 and baked at $80^{\circ} \mathrm{C}$ for 5 minutes, and then $250^{\circ}$ for one hour, according to manufacturer's specifications. The resulting polyimide alignment layers were rubbed with a commercial rubbing cloth (YA-20-R, Yoshikawa Chemical Co.) on a Optron rubbing machine with a roller radius of $r=4 \mathrm{~cm}$ rotating at $\sim 1000 \mathrm{rpm}$ while the sample was translated underneath at $\sim 1 \mathrm{~mm} \mathrm{~s}^{-1}$. This produced an easy axis $\hat{e}$ for planar liquid crystal alignment. For PVA substrates, a $0.5 \%$ by weight PVA $\left(\mathrm{M}_{\mathrm{w}}=\right.$ $85,000-124,000)$ in water mixture was spin coated onto the reflective substrate at $3000 \mathrm{rpm}$ for $30 \mathrm{~s}$. The PVA coated substrate then was baked at $120^{\circ} \mathrm{C}$ for $120 \mathrm{~min}$, allowed to cool, and then rubbed in the same manner as the RN1175 samples using a separate track of the rubbing cloth. In order to obtain a uniform thin film, a solution of liquid crystal in solvent was spin coated onto the substrates. A $0.2 \mathrm{M}$ solution of 9004 in toluene was prepared, and approximately $40 \mu \mathrm{L}$ of this isotropic solution was deposited on a substrate at room temperature. The substrate was then rotated for $30 \mathrm{~s}$ at rotational velocities between 1500 and $2500 \mathrm{rpm}$. This spin coating method evaporated the solvent and left a relatively uniform layer of liquid crystal, comparable to that obtained on $\mathrm{MoS}_{2}$. In order to vary the film thicknesses with this method, the samples were spun at higher or lower velocities. The thickness of the liquid crystal films for all substrates was estimated by their interference colors using polarized reflection microscopy ${ }^{23,24}$. Referring to a Newton tint table [Ref. 23,24], we were able to estimate the local thickness to within $\pm 15 \mathrm{~nm}$ for the thickness range 70 to $350 \mathrm{~nm}$.

\subsection{Imaging}

The samples were heated into the isotropic phase and then cooled to the nematic phase to facilitate orientational alignment. The samples then were slowly cooled in intervals of $0.1^{\circ}$ from the nematic phase into the Sm-A phase, and eventually into the $\mathrm{Sm}-\mathrm{C}$ phase. The temperature was allowed to stabilize for each temperature step, after which polarized micrographs were taken at (at each temperature step) using a 
high resolution camera (23 megapixels) and a Olympus BX60 polarizing microscope in reflection mode between crossed polarizers. The long-working-distance 50x objective lens had a numerical aperture NA $=0.85$, which facilitated good temperature control but resulted in an Airy diffraction diameter $\sim 065 \mu \mathrm{m}$. For maximum contrast, the samples were usually positioned with their easy axes set at $\sim 45^{\circ}$ with respect to the polarizer direction. These images were taken the same day as the samples were fabricated to minimize any possible degradation of the samples - or absorption of liquid crystal into the substrates - with time.

\section{Results and Discussion}

\section{1 $\mathrm{MoS}_{2}$ substrates}

Thin film Sm-A oily streaks ${ }^{7,12}$ were observed on cooling the liquid crystal into the Sm-A phase, as shown for $\mathrm{MoS}_{2}$ in Fig. 3a. On cooling further toward the Sm-A - Sm-C transition temperature $T_{A C}$, the formation of fan shaped focal conic domains ${ }^{32}$ was observed in regions where there previously had been oily streaks. These domains appeared at the thickest regions $(\sim 350 \mathrm{~nm})$ of the liquid crystal films. In thinner regions, as the temperature was decreased further, they evolved into regular arrays of streaks parallel to the easy axis. Figure $3 \mathrm{~b}$ shows the initial formation of these new structures, which we refer as "soapy streaks", as their formation apparently eliminates optical evidence of the oily streaks. With decreasing temperature, the soapy streaks were found to grow

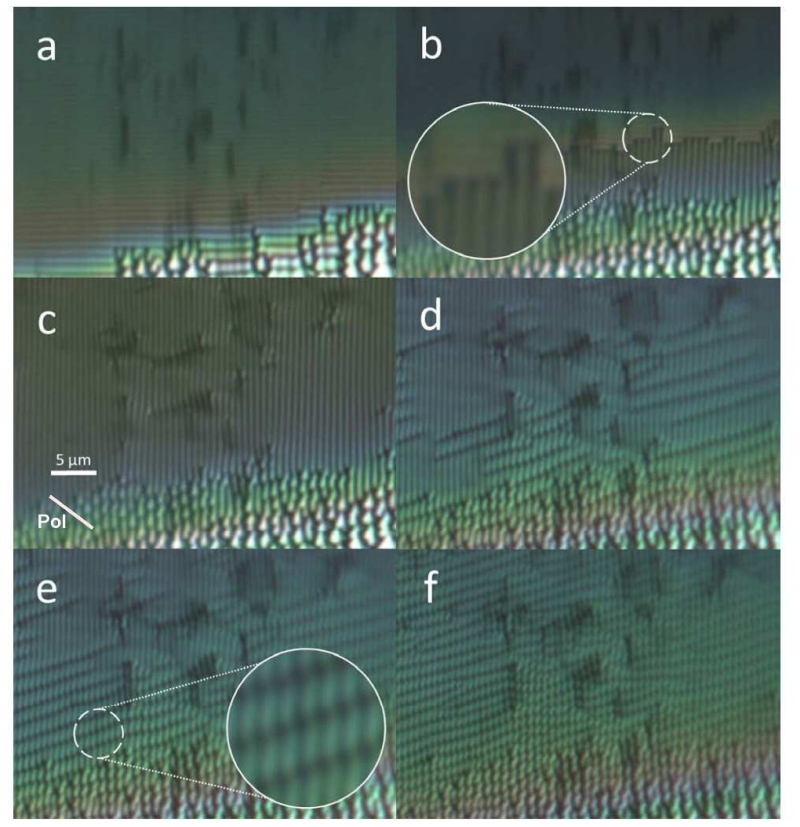

Fig 3. Photomicrographs of 9004 thin films showing soapy streaks on $\mathrm{MoS}_{2}$ at decreasing temperature, with $\mathrm{T}_{\mathrm{AC}} \mathrm{T}$ : a) $\left.0.1 \mathrm{~b}\right) 0.3$, c) 0.5 , d) 6.3 , e) 6.6 and f) $7.6 \mathrm{~K}$. Inset in (b) shows the discrete growth of the soapy streaks, and the inset in (e) shows a magnified view of soapy streak undulations. The $5 \mu \mathrm{m}$ scale bar in (c) is oriented perpendicular to the easy axis, and the bar labeled Pol corresponds to the direction of the polarizer. Film thickness is $300-350 \mathrm{~nm}$. discontinuously into the regions where oily streaks had been observable in the Sm-A phase. As the temperature was reduced deeper into the film's Sm-C phase, the soapy streaks suddenly - that is, in a time not resolvable by a 30 frames per second camera - extend to the dark region of the next oily streak, growing in an incremental fashion, as is apparent in the inset of Fig. 3b. More specifically, as the soapy streaks formed, their progress always began and terminated in the darkest region of the oily streaks. In the heretofore current model of oily streaks, these darker regions are believed to be characterized by a mostly vertical director orientation [Ref. 12, region D of Fig. 1], except near the liquid crystal / alignment layer interface. This discrete growth of soapy streaks occurred in a reversible and quantized manner, and the growth length in each step corresponded to the width of the oily streaks through which they propagated. While groups of adjacent soapy streaks generally progressed as a uniform front, they also could be found to progress individually, with one or more streaks outpacing the group by multiple oily streak widths.

With a further decrease in temperature inside the Sm-C phase, the field of view became completely covered with soapy streaks (Fig. 3c). Upon continued reduction of the temperature, the soapy streaks began to undulate in the plane, with an undulation periodicity larger than the width of the streaks themselves, (shown in Figs. 3d, 3e, and 3f). The waviness of adjacent streaks appeared to be correlated, as nearby streaks tended to undulate together, forming a texture of repeating waves in which the phase varied nearly regularly from streak to streak (inset, Fig. 3e). 
In addition to 9004, it was possible to reproduce qualitatively these behaviors with other liquid crystals. Most

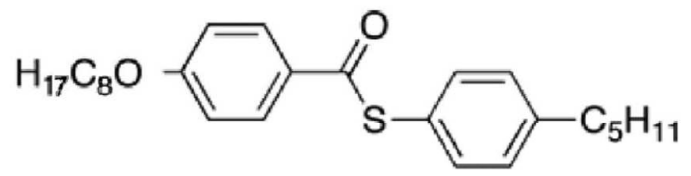

Fig. 4 Molecular structure of liquid crystal $\overline{8} S 5$

notably, we found similar behavior for the liquid crystal 4-npentylphenylthiol-4'-n-octyloxybenzoate $(\overline{8} S 5$, Fig. 4 , phase sequence Iso - 86 - Nematic - 64 - Sm-A - 56 - Sm-C - $30.6-$ Sm-J - 14.6 - Crystal) for both oily and soapy streaks, as shown in Fig. 5. Like $9004, \overline{8} S 5$ has a Sm-A $-\mathrm{Sm}-\mathrm{C}$ phase transition. This is unlike observations of oily streaks in the Sm-A phase using the liquid crystal octylcyanobiphenyl $(8 \mathrm{CB})$, which does not exhibit a Sm-C phase. One difference between the $\overline{8} S 5$ and 9004 systems should be noted: For larger film thicknesses (> $350 \mathrm{~nm}$ ), films of 9004 no longer exhibit soapy

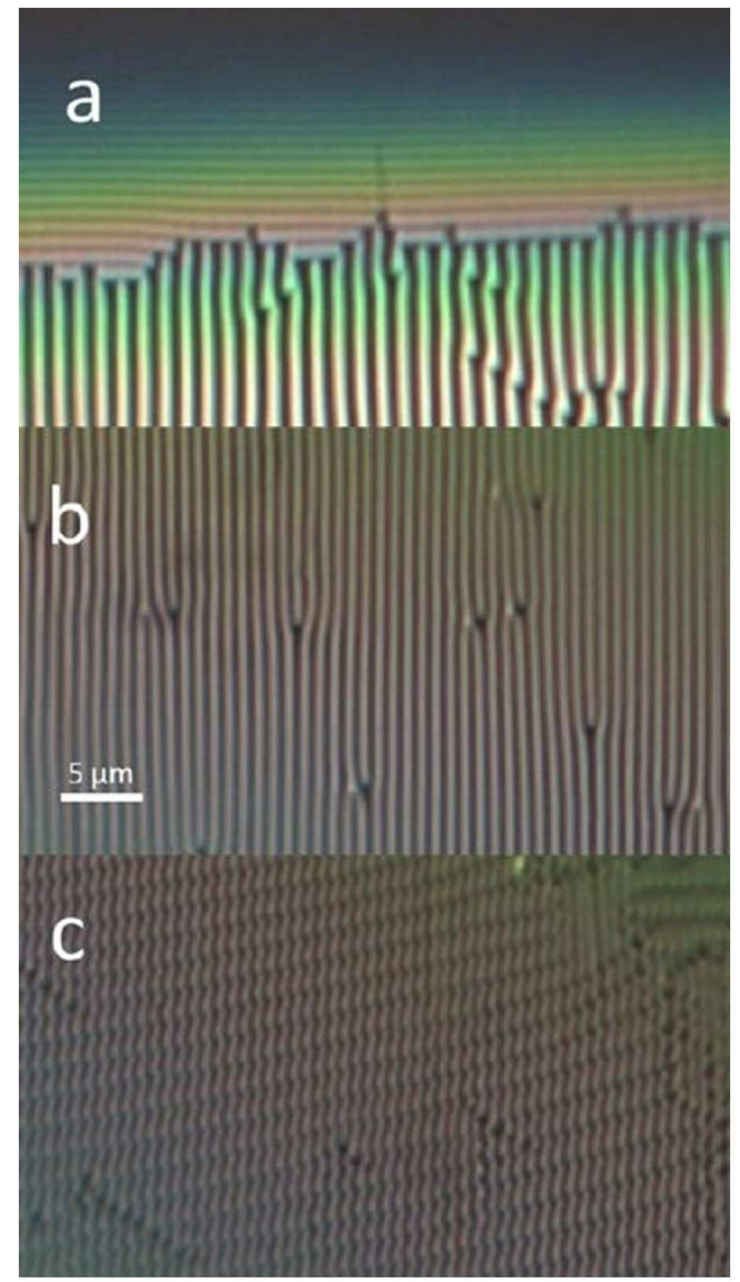

Fig. 5 Photomicrographs of $\overline{8} S 5$ soapy streaks on $\mathrm{MoS}_{2}$ at $T_{A C}-T \sim$ a) 0.1 b) 3.1 and c) $8.5 \mathrm{~K}$. The scale bar is oriented perpendicular to the easy axis and the polarizer is at $45^{\circ}$ with respect to the scale bar. Film thickness is $\sim 300 \mathrm{~nm}$. streaks, but instead transitioned directly to focal conics. This is not the case for $\overline{8 S 5}$, as soapy streaks appear in much thicker films of at least up to $450 \mathrm{~nm}$.

\subsection{Polyimide and PVA}

Similar to oily streaks, soapy streaks were observed to form on a variety of substrates, such as PVA, RN1175, and $\mathrm{MoS}_{2}$. However, there were some noticeable differences among the soapy streaks as they developed on these different substrates. When 9004 was deposited onto $\mathrm{MoS}_{2}$, the streaks and patterns tended to be relatively uniform, with regular linear arrays at higher temperatures, transforming to uniform undulations and sawtooth patterns at lower temperatures. The difference between the soapy streaks on different substrates was especially noticeable once the undulating patterns had formed. The undulations on $\mathrm{MoS}_{2}$ had a uniform periodicity, giving the wavy texture a quasi-long range order (Figs. 3e and 3f). On RN-1175 - as well as on PVA - the undulations of a given soapy streak sometimes showed far less correlation with its neighbors; the left-hand side of Fig. 6c shows an example of this. This difference likely can be explained by the nature of the alignment layers: While $\mathrm{MoS}_{2}$ aligns on the atomic level and is atomically flat, polyimide

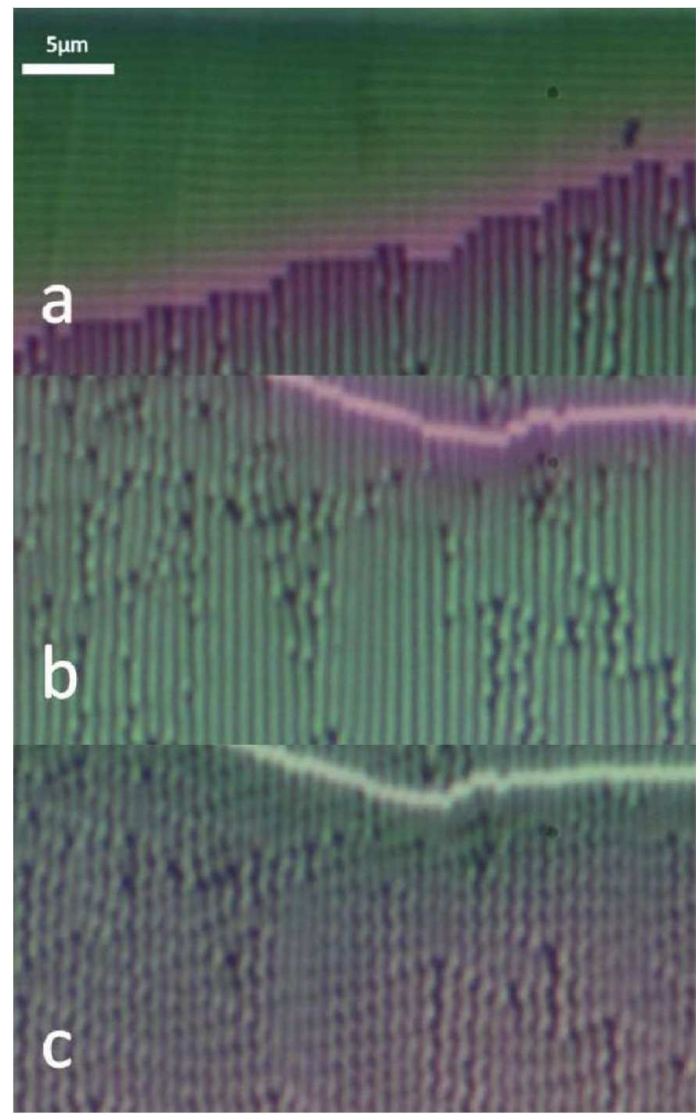

Fig. 6 Photomicrographs of soapy streaks that formed from 9004 on RN1175 at $T_{A C}-T$ a) 0.1, b) 4.3 , and c) $7.4 \mathrm{~K}$. All images were taken at the same location. The $5 \mu \mathrm{m}$ scale bar is oriented perpendicular to the easy axis and the polarizer is at $45^{\circ}$ with respect to the scale bar. Film thickness is $\sim 250 \mathrm{~nm}$. 
alignment layers are comparatively rough and rely on external rubbing to create an easy axis. This external rubbing gives a macroscopically uniform easy axis; however, there can be some variation on length scales comparable to the period of oily and soapy streaks. The roughness and microscopic variations very likely could contribute to the formation of the less ordered soapy streaks on polyimide and PVA.

\subsection{Contrast Measurements}

To study the evolution of reflected light intensity in the bright and dark regions of the soapy streaks, photographs were taken in reflection mode of the streaks oriented at various angles with respect to the polarizer and crossed analyzer, and were imported into the photo analysis software Image- - . Intensity profiles along an axis perpendicular to the soapy streaks were extracted for each angle $\beta$, and plotted vs. their position (perpendicular to the soapy streaks); the data are shown in Fig. 7. An apparent spatial periodic intensity dependence of contrast can be observed (as already seen in Figs. 3 and 5) - the contrast is partially reduced by diffraction effects due to the microscope optics - but this is not the most interesting feature. It should be noted that whereas the oily streaks always become uniformly and nearly completely dark when they are parallel to either of the crossed polarizers this can be seen in Fig. 8 - the soapy streaks remain visible, independent of the orientation of the easy axis with respect to the polarizers, even at $\beta=0^{\circ}$. The soapy streaks also show larger contrast than the oily streaks, as is apparent from Fig. $5 a$ in regions of the cell where soapy streaks and oily streaks have the same thickness. When oily streaks are imaged between crossed polarizers, it is the change from mostly homeotropic alignment in the dark regions to tilted - or even nearly planar - alignment in the brighter regions that provide their variations in contrast at angles $\beta$ other than $0^{\circ}$ or $90^{\circ}$ with respect to the polarizers. Because the director always lies in a

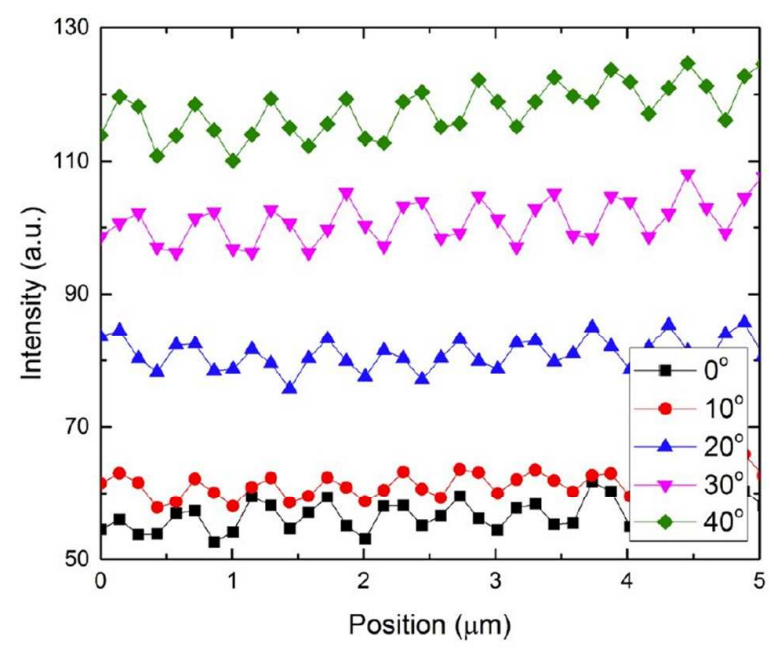

Fig 7 Intensity vs. position plot for a series of soapy streaks (9004 on $\mathrm{MoS}_{2}$ ), along an axis perpendicular to the soapy streaks, i.e., perpendicular to the easy axis, vs. angle $\beta$, which is the sample rotation angle with respect to the crossed polarizers. Lines are meant as a guide to the eye. Data collected at $\mathrm{T}_{\mathrm{AC}}-\mathrm{T} \sim 1 \mathrm{~K}$.

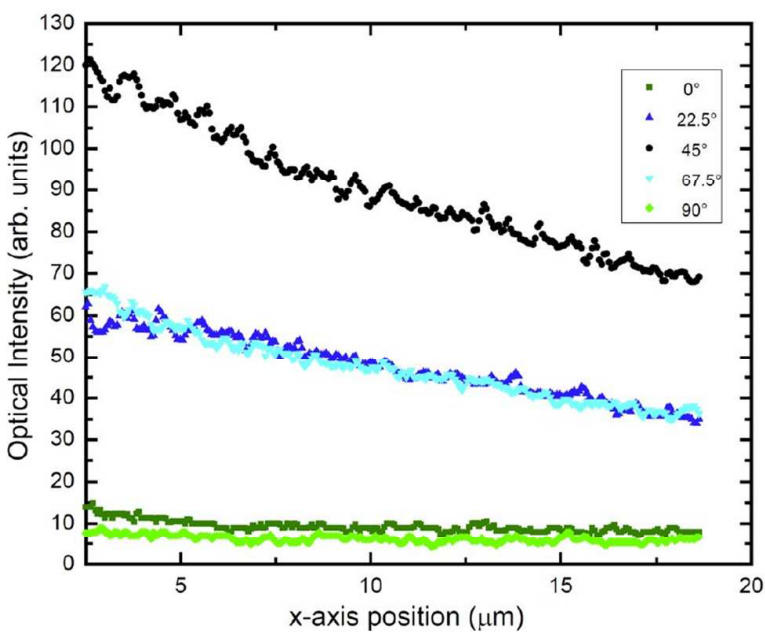

Fig. 8 Intensity vs. position along an axis perpendicular to the oily streaks, i.e., parallel to the easy axis, for a series of oily streaks (9004 on $\mathrm{MoS}_{2}$ ) oriented at several angles $\beta$ with with respect to the crossed polarizers. The slow decrease of intensity with position is due to a variation of film thickness over this region. Notice the weak contrast (the periodicity of the intensity) even at $\beta=45$, and that the oily streaks can be nearly extinguished for $\beta=0^{\circ}, 90^{\circ}$. Data collected at $T-T_{A C}$ $\sim 2 \mathrm{~K}$.

plane perpendicular to the oily streaks, oily streaks exhibit a maximum of extinction when the streaks are oriented parallel to either polarizer. Since this is not the case for the soapy streaks, the cause must be a variation of the director alignment way from the homeotropic to planar tilt plane. Since the soapy streaks are formed in the $\mathrm{Sm}-\mathrm{C}$ phase, it seems clear that the $c$-director (the projection of the molecular director into the smectic layer plane) must be involved in their formation. However, the small birefringence associated with the tilt of the molecules, particularly in 9004 (with a very small tilt angle $\sim 3^{\circ}$ as measured by $x$-rays in the Lemieux lab) does not provide sufficient optical retardation to explain directly the optical data in Fig. 7. We argue instead that complicated boundary conditions for the $\mathrm{Sm}-\mathrm{C}$ director result in disclinations, which in turn change details of the patterns of the smectic layer normal, resulting in the relatively high contrast of the soapy streaks. This will be detailed below.

3.4 X-ray Measurements 
In addition to optical experiments, transmission small angle $x$-ray scattering (TSAXS) measurements have been performed $^{33}$, the results of which are summarized here. Samples of thin 9004 films on polyimide coated glass slides were prepared and confirmed by optical microscopy to exhibit both oily streaks in the Sm-A phase as well as soapy streaks in the $\mathrm{Sm}-\mathrm{C}$ phase. Experiments were performed at the ESRF (Grenoble-France) synchrotron on the D2AM beamline. For this set of measurements the beam energy was set to $20 \mathrm{keV}$ with a transverse dimension of $300 \times 300 \mu \mathrm{m}^{2}$. We worked in the transmission geometry with the sample positioned such that the substrate was located on the incident beam side. The scattered signal was recorded with a $2 \mathrm{D}$ detector. When the sample was initially oriented perpendicular to the $\mathrm{x}$-ray beam, two symmetric scattered arcs at wavevector $Q=0.22 \mathrm{~A}^{-1}$ were observed. They are associated with the $\mathrm{x}$-ray scattering from smectic layers perpendicular to the substrate and perpendicular to the easy axis; the latter are parallel to the polyimide rubbing, and are observed (Figure 9). For the Sm-A phase (Figure 9a) the $x$-ray arc/extension in the $Q_{y}$ direction remains limited (Figure 9a) and is similar to that observed in the Sm-A phase of $8 \mathrm{CB}$ with oily streaks ${ }^{16}$. For the $\mathrm{Sm}-\mathrm{C}$ phase, the scattered arc maintains the same position on the $2 \mathrm{D}$ detector, in agreement with a very small tilt angle $\sim 3^{\circ}$, showing that a majority of smectic layers are still perpendicular to the easy axis. However the scattered arcs appear much more concentrated for the Sm-A phase than for the Sm-C phase, where the arc extension in the $Q_{y}$ direction is larger (Fig. 9a). This suggests that, unlike in the Sm-A phase, a significant number of smectic layers have their layer normal lie outside the plane defined by the substrate normal and the easy axis direction. This inference is in agreement with the non-zero optical microscopy contrast of the soapy streaks when $\beta=0^{\circ}$ (Figure 7). One must account for this observation in any model of the soapy streak structure.

We then rotated the sample, which allows for a tilt of the wavevector transfer with respect to the substrate plane, the

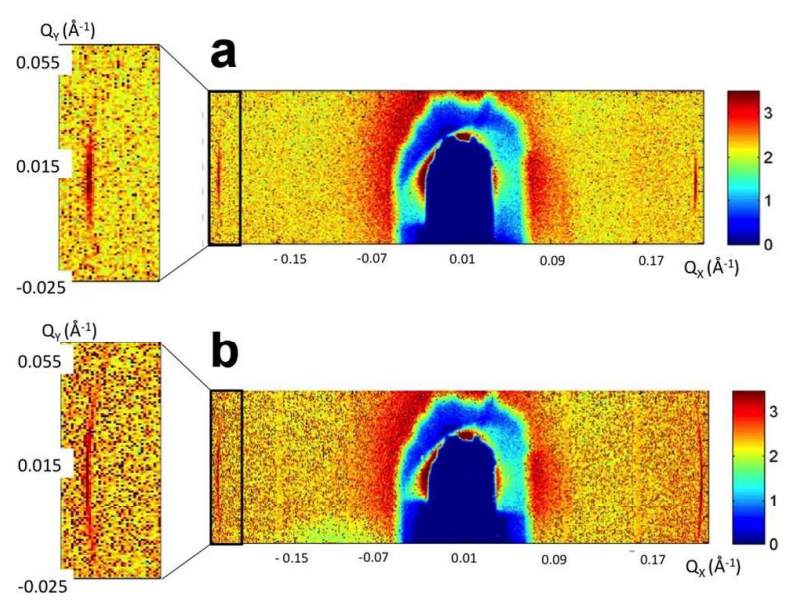

Fig. 9 Scattered $x$-ray signal of the 9004 smectic layers on rubbed polyimide (thickness between 150 and $250 \mathrm{~nm}$ ) in (a) Sm-A phase and (b) Sm-C phase. Enlargements of the scattered signals are shown at the left of each image. The axes represent the scattered wavevector in units of $\AA^{-1}$. The colors correspond to a logarithmic scale of the $x$-ray intensity at the $2 \mathrm{D}$ detector. Data collected at approximately $2 \mathrm{~K}$ above $\mathrm{T}_{\mathrm{AC}}$ (a) and $2 \mathrm{~K}$ below $\mathrm{T}_{\mathrm{AC}}(\mathrm{b})$.
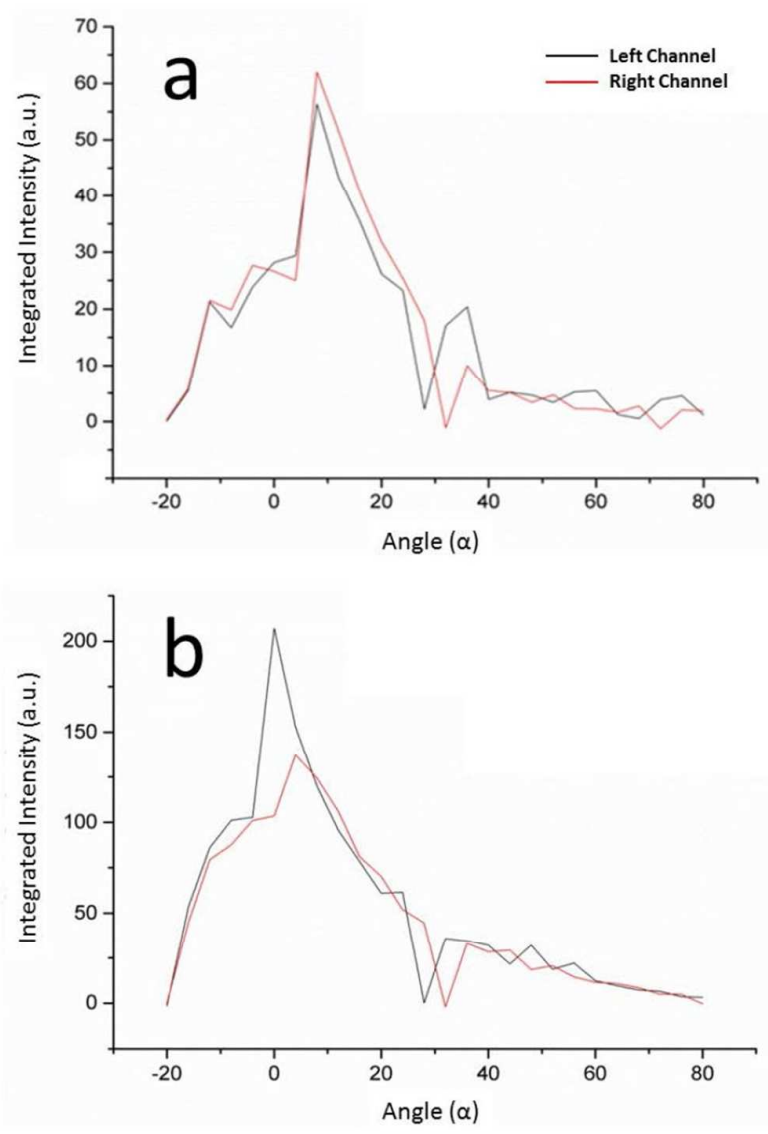

Fig. $10 \mathrm{X}$-ray scattering data showing the integrated scattering intensity vs. smectic layer angular distribution for a) Sm-A oily streaks in a $100-130 \mathrm{~nm}$ film and b) Sm-C soapy streaks in a $160-180 \mathrm{~nm}$ film. Notice the $10^{\circ}$ angular offset in the Sm-A phase, and the broadening of the angular distribution in the $\mathrm{Sm}-\mathrm{C}$ phase.

tilt being defined by the angle $\alpha$. In Fig. 10 we plot the evolution of the integrated arc intensity after subtraction of the background diffusion as a function of the angle $\alpha$ (Fig. 10a for Sm-A phase and Fig. $10 \mathrm{~b}$ for Sm-C phase). The integrated intensity is proportional to the number of layers oriented perpendicular to the wavevector transfer. The distribution orientation of the layers is thus followed by the intensity value with respect to the rotation of the sample with respect to the incident beam. Figure 10a presents such an evolution for the Sm-A phase. One observes a typical variation, similar to those that have been reported in previous works on $8 \mathrm{CB}$ oily streaks ${ }^{14,16}$. The $10^{\circ}$ shift of the intensity maximum from $\alpha=$ $0^{\circ}$ in the Sm-A phase is attributed to a molecular pretilt - and therefore a tilt in the smectic layers - at the polyimide substrate. This already has been observed for $8 C B$ on polyimide substrate and is absent on PVA substrate ${ }^{16}$. Moving to the $\mathrm{Sm}-\mathrm{C}$ results presented in Fig. 10b, we notice some differences. Most notably, the maximum of intensity is now around $\alpha=0^{\circ}$ rather than $10^{\circ}$. This shift is in agreement with a transition from the Sm-A to Sm-C phase. Apart from this, the data for the Sm-A and Sm-C phases are quite similar, the main difference corresponding to an overall smaller intensity for the 
smectic A phase. This could be due to a smaller thickness for the probed smectic A phase area, or to a larger number of bent smectic layers in smectic $\mathrm{C}$ phase with respect to the flat or quasi-flat smectic layers.

\section{Proposed Model of the Structure}

With the available data on the nature and appearance of these soapy streaks, a description of the structure is beginning to emerge. Here we present a speculative but reasonable scenario for the development of the soapy streaks that accounts for our experimental observations.

To begin building a model for soapy streaks, we return to the oily streak model. For reference, consider the cartoon of a thin film oily streak's cross section above the planar transition region (Fig. 1 region E) in the xz-plane, which is shown in Fig. 1. This picture is based on microscopy, ellipsometry (of oily, not soapy, streaks), and X-ray data of $8 \mathrm{CB}$ on PVA and $\mathrm{MoS}_{2}{ }^{7,15,16}$, and is consistent with the boundary conditions at the two interfaces. This oily streak picture may now be modified slightly in order to facilitate a transformation to soapy streaks in the Sm-C phase, while still being consistent with the experimental results characterizing the oily streaks. In particular, the model pictured in Fig. 1 has a large region in which the smectic layers are represented as being flat (item D) and also possesses a thin region over the entire substrate where the smectic layer normal is parallel to the substrate's easy axis. Instead, we shall now introduce a small amount of curvature (to remain consistent with $\mathrm{x}$-ray data) in what had been the flat smectic layers, as shown in Fig. 11a. Although flat layers are a useful device for modeling the X-ray data, they would have a constant free energy per unit length. Thus, over long distances compared to the interaction lengths, flat layers are inconsistent with a fixed period determined by interactions. While the smectic layers will be mostly nearparallel to the substrate close to the center of the unit cell / optically dark region of the oily streak, the boundary condition at the substrate has the nematic director lie in the plane of the substrate. Thus, in this region (shown in red in Fig. 11a, and region E of Fig. 1) close to the unit cell center and to the substrate, there must be a large bend of the layer normal over a small distance. Another possibility would be melting of the liquid crystal into the nematic phase ${ }^{16,34}$ so that smectic layering vanishes and only the nematic director has to bend; this scenario would occur in conjunction with a $+1 / 2$ strength disclination at the center (Fig. 11a). We further posit that the layer normal above the red region of Fig. 11a, i.e., the director $\widehat{n}$, becomes less parallel to the substrate normal (z-axis) as one moves away from the center of the unit cell. Not shown in Fig. 11a are the smectic layers near the substrate, which form the remainder or "other side" of this extended-core disclination (region A of Fig. 1), and the rotating grain boundary (region E of Fig. 1). In the Sm-A / oily streak phase, because the nematic director, which also is the layer normal, resides only along the $x z$-plane, there can be disclinations of any half-integer strength. Moreover, the sum of all these defect strengths in a unit cell must be zero. Thus, in this picture, there is one $+1 / 2$ disclination and one $-1 / 2$ disclination in each unit cell. We will refer to the mirror plane through the $+1 / 2$ disclination (the red dot in Fig. 11a, and the center of the optically dark part of the oily streak) as the "center" of the unit cell, and the mirror plane through the $-1 / 2$ disclination (the blue dot in Fig. 11a and the optically bright part of the oily streak) as the "edge" of the unit cell. The $+1 / 2$ disclination requires one region (shown in red in Fig. 11a) with concentrated curved smectic layers (shown as green lines), which occurs near the substrate at the

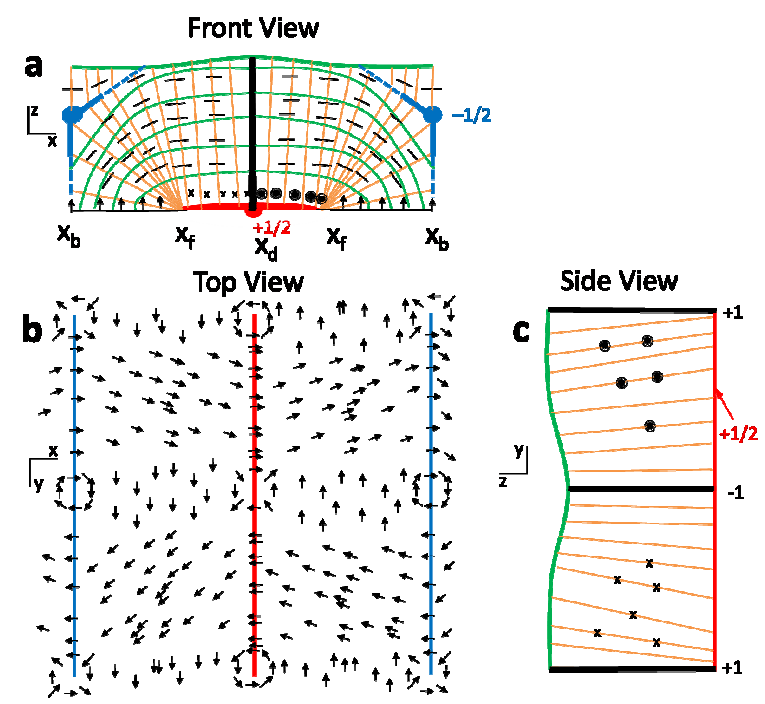

Fig. 11 Modified view of oily streaks and development of soapy streaks. To decrease the space required for a legible figure, the $z$ axis of ( $a$ and c) has been expanded. For the soapy streaks in the Sm-C phase, the y axis of figure (b) has been compressed. a) Front view cross section of proposed oily streak structure in the $\mathrm{Sm}-\mathrm{A}$ and the $\mathrm{Sm}-\mathrm{C}$ phases, where the oily streaks run perpendicular to the $\mathrm{xz}-$ plane. The period (along the $x$-axis) of the Sm-A phase oily streaks is $\sim 1-2 \mu \mathrm{m}$, but in the $\mathrm{Sm}-\mathrm{C}$ phase the oily streak period is smaller than optical wavelengths. The soapy streaks ultimately run approximately parallel to the $x$-axis. We also point out few smectic layers above $-1 / 2$ disclinations may exist at the air interface. b) A top view looking down at the cell. c) Side view (the $y$-z plane) in which one of the +1 disclination lines passes perpendicular to the plane in (a) through the unit cell center. Thus the red-colored base of (c) corresponds to the $+1 / 2$ disclination shown by the red dot in (a). General symbols: The green lines correspond to smectic layers; the orange lines correspond to the smectic layer normal; the red and blue dots correspond to an end-on view of director disclination lines, and the red and blue lines correspond to a side view of these disclination lines; the black arrows, encircled dots (arrow heads) and x's (arrow tails), and line segments represent the preferred direction of the pseudovector order parameter $\vec{\theta}$ in this structure, which characterizes the molecular tilt when the temperature falls below $\mathrm{T}_{\mathrm{AC}}$. The thick black lines in (a) and (c) correspond to integer disclination defects, which can be seen in the top view (b). Finally, everywhere on the upper surface, the projection of the layer normal (not shown) onto substrate is rotated clockwise by $90^{\circ}$ with respect to the black arrows that represent the pseudovector $\vec{\theta}$ in (c)

bottom of the figure. The $-1 / 2$ disclination could, in principle, be a single region of director bend with the angle of bend varying from $0^{\circ}$ to some large angle, which would necessitate the existence of a few smectic layers above the curvature wall (Fig. 1 region C). These layers would provide only a minimal contribution to the $\mathrm{x}$-ray profiles. However, it is almost certain that the free energy is lower if, instead, there were three 
distinct regions, with the orientation in each going from 0 to $\pm 30^{\circ}$, consistent with the blue lines in the schematic drawing. The precise structure would depend on the thickness of the film.

The two sets of $\mathrm{x}$-ray data corresponding to the oily (Sm-A phase) and soapy ( $\mathrm{Sm}-\mathrm{C}$ phase) streaks, are quite similar. This suggests that, rather than transitioning into a completely new structure, the transition from oily to soapy streaks is associated with a modification of the pre-existing structure. We believe that the disappearance of the light modulation due to oily streaks and the concomitant appearance of the soapy streaks is caused by the formation of smectic-C order. As noted above, $x$-ray experiments clearly show that the distribution of angles of smectic layers is not dramatically different between the soapy and oily streak structures, indicating that the oily streaks do not disappear. The fact that oily streaks are not observed optically suggests that the period of the "modified oily streaks", i.e., the underlying oily streaks below $\mathrm{T}_{\mathrm{AC}}$, becomes smaller and cannot then be observed by visible light. This would correspond to an increase in the number of curved layers with respect to the quasi-flat layers, in agreement with the x-ray data shown in Figure 10. Thus, we now use Fig. 11 to represent a speculative modification of our previous picture of the oily streak. In particular, Fig. 11a corresponds generically to both the "regular" long period Sm-A oily streaks (Fig. 1) and to the "modified" short period Sm-C oily streaks

This change of period is caused by the Sm-C elastic energy $^{35,36}$, together with the boundary conditions on the director, as described below. However, these boundary conditions on the director in the $\mathrm{Sm}-\mathrm{C}$ phase result in a new texture in which there is, perpendicular to these short period oily streaks, a spatially periodic array of ordinary disclinations of the director having strength \pm 1 (see below). The optical modulation associated with the soapy streaks likely is caused by the effect these disclinations have on the equilibrium pattern for the layer normal. The rather small $\left(\sim 3^{\circ}\right)$ director tilt with respect to the smectic layer normal and the resulting birefringence are very small and do not plausibly provide the observed optical contrast (including the reduction of observed contrast by diffraction limited effects) seen in Figs. 5, 6, 7, and 8. However, it is reasonable to assume that the fraction of layers that are not planar (evidenced by combined optical microscopy and x-ray diffraction) differs depending on the location relative to the disclinations, resulting in the soapy streak modulation. How might this happen?

Let us consider the consequences of this oily streak structure for the $\mathrm{Sm}-\mathrm{C}$ phase. To avoid confusion associated with the sign of the smectic layer normal, we will characterize the molecular orientation in the $\mathrm{Sm}-\mathrm{C}$ director by a pseudovector $\vec{\theta}$. This pseudovector, $\overrightarrow{\theta \equiv \widehat{N} \times \vec{c}}$ (to within a chosen chiral proportionality constant), is in the direction of the vector that would correspond to the polarization vector if the liquid crystal were a ferroelectric chiral Sm-C. Here $\widehat{N}$ is the smectic layer normal (the orange lines in Figs. 11a and 11b) and $\vec{c}$ is the projection of the long axis of the molecule onto the smectic layer plane. Thus $\vec{\theta}$ is a pseudovector in the plane of the smectic layers, and is shown by the black arrows in Figs. $11 \mathrm{a}, 11 \mathrm{~b}$, and 11c, including the arrowheads represented by the encircled black dots and arrow tails by the symbol " $x$ ". At the substrate, where the nematic director or smectic layer normal $\widehat{N}$ is nearly parallel to the substrate, there is a sharp curvature of the layer normal going away from the substrate, or from the underlying planar oriented liquid crystal, into the bulk smectic. This occurs especially in the red regions just above the substrate, where the orange lines, corresponding to $\widehat{N}$, must curve dramatically. This distortion has the same symmetry as the $\mathrm{Sm}-\mathrm{C}$ pseudovector order parameter, where a linear coupling between the torque on the layers and the $\vec{\theta}$ director is expected. Thus the Sm-C parameter $\vec{\theta}$ points perpendicular to the plane of the picture (the dots and $x^{\prime} s$ ), and in opposite directions near the center of the unit cell. At the substrate near the edges of the unit cell, where there is planar alignment and the smectic layer normal lies in the plane of the substrate, the lack of symmetry of the substrate suggests that $\vec{\theta}$ should be along the $z$ axis. On an achiral substrate like PVA there should be no distinction between $\vec{\theta}$ parallel or anti-parallel to $\hat{z}$; whereas on a chiral substrate like $\mathrm{MoS}_{2}$, either parallel and antiparallel to $\hat{z}$ will be preferred. Finally, in the rest of the cell, the elastic constants of the Sm-C phase ${ }^{39,40}$ will have a tendency to align $\vec{\theta}$ so that it is either parallel or perpendicular (with no particular sign) to layer normal splay, and also parallel or perpendicular to layer normal bend, which will be concentrated in grain boundaries. These are shown by the short black lines in Fig. 11a. Unfortunately the relevant $\mathrm{Sm}-\mathrm{C}$ elastic constants, and even their signs, are unknown. Nevertheless, it is reasonable that the pseudovector $\vec{\theta}$ prefers to be in the plane in which the layer normal varies, that is to say, in the plane of drawing in Fig. 11a.

Finally, i) following Mermin ${ }^{37}$, ii) given the $C_{2 h}$ symmetry of the $\mathrm{Sm}-\mathrm{C}$ phase, and iii) as the layer normal varies in space, we can conclude that there are three possible disclinations in the $\mathrm{Sm}-\mathrm{C}$ phase with strengths $+1 / 2,-1 / 2$ and \pm 1 , provided that the $\vec{\theta}$ pseudovector can orient out of the xz-plane of Fig. 11a. Figure 11c shows the yz-plane that passes through the unit cell center in Fig. 11a, including the \pm 1 strength disclinations (the large black dots). These disclinations have the additional requirement that they must add modulo 2 . A strength $+1 / 2$ or $-1 / 2$ layer normal disclination also must be either $a+1 / 2$ or $a-$ $1 / 2$ Sm-C disclination. However, contrary to usual expectations, the integer disclinations in the $\vec{\theta}$ director can have energies lower than the $+1 / 2$ or $-1 / 2$ disclinations: The $+1 / 2$ and $-1 / 2$ disclinations require expensive layer distortions while the integer disclinations do not.

These boundary conditions, which, if naively extrapolated from the substrate, result in oppositely pointing $\vec{c}$ directors "above" (i.e., closer to the air interface than to the substrate) the $+1 / 2$ and $-1 / 2$ disclinations. Given our data, it seems reasonable to suggest that the resulting elastic energy is relieved, in part, by the +1 and -1 disclinations that we suggest intersect $1 / 2$ strength disclinations.

Thus, owing to the hybrid anchoring conditions, the influence of the oily streak structure on the director in the Sm- 
$C$ phase has two consequences: First, the spacing of the oily streak unit cell along the $x$-axis decreases and becomes optically undetectable, at least in the visual part of the spectrum. Second, \pm 1 ordinary Sm C disclinations along the zaxis illustrated in Fig 11c are formed in order to relieve the rapid variation in the $\mathrm{Sm}-\mathrm{C}$ order parameter implied by the boundary conditions. As shown in Figs. 11a and 11b, we hypothesize that these are periodic along the $x$-axis, intersecting with the $1 / 2$ strength disclinations and also having the soapy streak periodicity along the $y$-axis. The presence of these disclinations changes the preferred layer normal direction and the resulting birefringence is observed as soapy streaks. Note also that this structure possesses the twodimensional space group P2, i.e., it is a periodic structure for which the point group is a two-fold rotation around the substrate normal. As such, it is consistent with the observed slow evolution to the lower temperature wavy structure, which has (only) this symmetry. The periodic waves are a consequence of the hidden / short period oily streaks in this phase.

This picture is at least qualitatively consistent with our $\mathrm{x}$ ray and optical data. The elongated Bragg spots observed in Fig. 9 show that the layer normal $\widehat{N}$ in the Sm-C can have significant tilt out of the xz-plane. Near the integer strength disclinations $\widehat{N}$ is vertical at the center and tilts in effectively all directions around the vertical, although in other parts of this structure the tilt is primarily in the $\mathrm{xz}$-plane, i.e., the plane including the substrate normal and the rubbing direction. Thus, when the angle between the polarizer / analyzer and the rubbing direction is close to $45^{\circ}$, the region where there are disclinations is dark, as the tilts at $45^{\circ}$ to the rubbing direction do not contribute to the birefringence / retardation. On the contrary, when the angle between the polarizer / analyzer and the rubbing direction is close to $0^{\circ}$ and $90^{\circ}$, the important retardation comes from the tilt of $\widehat{N}$ that is neither parallel nor perpendicular to the rubbing direction, e.g., close to at $45^{\circ}$ to the rubbing direction. These tilts are concentrated near the disclinations, consistent with the experimental observation that the light and dark regions reverse for these two different polarizer/analyzer directions. Finally the complicated tilt structure is consistent with the observation that there is no orientation of the polarizer and analyzer for which the soapy streak contrast vanishes, unlike the case of oily streaks in the Sm-A phase (cf. Figs. 7 and 8). Thus, the general structure of the soapy streaks presented in Fig. 11 is consistent with the optical results in Figs. 5, 6, 7, and 8. In particular, the shorter period Sm-C phase "modified" oily streaks that retain their underlying structure are not visible at optical wavelengths. Moreover, the higher contrast of the soapy streaks relative to the oily streaks, and the observation that the soapy streak intensity cannot be made very small with rotation by angle $\beta$ (as it can for oily streaks), do not depend on the relatively small molecular tilt angles - and the resulting very small optical retardation - associated with the Sm-C phase. Rather, these features come about because of the \pm 1 strength disclinations (Figs. $11 \mathrm{~b}$ and 11c). Finally, the apparent stepwise elongation of the soapy streaks is related to the formation of disclinations and the concomitant decrease in the periodicity of the now-modified oily streaks.

Finally, we make a few general remarks about the wavy soapy streaks and sawtooth pattern observed on the $\mathrm{MoS}_{2}$ surface (Fig. 12). Scanning tunneling microscopy measurements of the liquid crystals $8 \mathrm{CB}^{16}$ and $5 \mathrm{CB}, 8 \mathrm{CB}, 10 \mathrm{CB}$ and $11 \mathrm{CB}^{38}$ on $\mathrm{MoS}_{2}$ reveal an arrangement of the surface layer of liquid crystal molecules that is chiral in two dimensions ${ }^{43}$. (The chirality actually is three-dimensional, but is localized very close to - within a few nanometers of - the surface.) Interestingly, the soapy streaks' sawtooth pattern observable for both 9004 and $\overline{8} S 5$ on the $\mathrm{MoS}_{2}$ substrate also is two-dimensionally chiral ${ }^{39-41}$. Moreover, the $2 \mathrm{D}$ chiral sawtooth pattern is not observed on the achiral polyimide and PVA alignment layers. Figure 12 presents an image of 9004 on $\mathrm{MoS}_{2}$, where the sawtooth shaped soapy streaks clearly exhibit two distinct chiral domains that are divided by a boundary on a single sample. Note that both 9004 and $\overline{8} S 5$ are configurationally achiral but conformationally racemic molecules with low energy barriers of order $0.8 \mathrm{k}_{\mathrm{B}} \mathrm{T}$ between

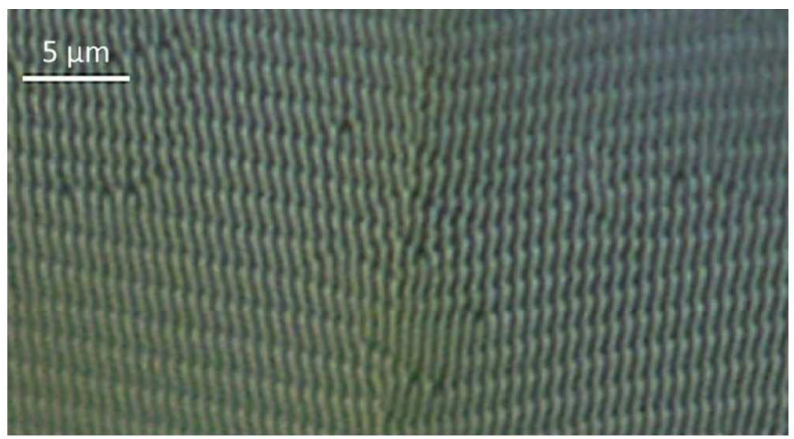

Fig. 12 Photomicrographs of undulating 9004 soapy streaks on $\mathrm{MoS}_{2}$ at $T_{A C}-T \sim$ $6.8^{\circ} \mathrm{C}$ exhibiting two distinct chiral domains. This image has been slightly contrast enhanced to aid the reader. The $5 \mu \mathrm{m}$ scale bar is oriented perpendicular to the easy axis and the polarizer is at $45^{\circ}$ with respect to the scale bar.

left and right handed conformers ${ }^{42}$. Here $k_{B}$ is Boltzmann's constant. This suggests that the surface chirality is being transmitted at least at small distance into the bulk, manifesting the macroscopic sawtooth-shaped streaks. This observation is consistent with the recent work of Basu, et al, who showed that flakes of graphene (which are similar in structure to $\mathrm{MoS}_{2}$ ) induce a measurable chiral signature when uniformly dispersed into a bulk achiral liquid crystal ${ }^{43}$. It is clear that considerably more work is required to elucidate the relationship between chirality and soapy streaks.

\section{Conclusions}

To summarize, we have presented observations of a new type of liquid crystal texture that likely occurs in the Sm-C phase in the presence of competing homeotropic and planar boundary conditions. Spawning from oily streaks in the Sm-A phase, these "soapy streaks" run orthogonal to the oily streaks and appear to elongate discontinuously from one oily streak to the next. At lower temperatures the soapy streaks begin to 
undulate and, interestingly, the undulations appear to be twodimensionally chiral for the $\mathrm{MoS}_{2}$ substrate. Combining the $\mathrm{x}$ ray results with the optical microscopy data collected on the soapy streaks, and accounting for the molecular tilt in the Sm$C$ phase, we have presented a possible scenario for the formation and structure of soapy streaks. This scenario suggests that the basic oily streaks structure is retained in soapy streak, but with a sharp decrease of the oily streak period and the implementation of periodic smectic $C$ disclinations perpendicular to the oily streaks. As a result the smectic-A / smectic-C phase transition leads to the transformation from a 1D network of topological defects into a 2D network.

\section{Acknowledgements}

We thank Andrew Ferris for assistance with oily streak intensity measurements and Dr. Laurent Pelliser with his help in the treatment of the x-ray data. IRN and CR were supported by the National Science Foundation's Condensed Matter Physics Program under grant DMR-1505389. EL was supported in part by UPMC-Matisse labex. RPL was supported by the Natural Sciences and Engineering Research Council of Canada. Travel between Paris and Cleveland and lodging were supported by the Partner University Fund, administered by the French Embassy in the United States. IG was supported by Campus France, Bourses d'Excellence Eiffel.

\section{Notes and references}

$\S$ The term "soapy streak" does not imply the existence of surfactants, and was chosen because they apparently cause the oily streaks to vanish visually.

1 P.G. De Gennes and J. Prost. The Physics of Liquid Crystals, Clarendon, Oxford, 1994.

2 M. G. Friedel. B1 Annales de Physique, 1922, 18, 273-474.

3 J. B. Fournier, I. Dozov, and G. Durand, Phys. Rev. A, 1990, 41, 2252-2255.

4 M. C. Choi, T. Pfohl, Z. Wen, Y. Li, M. W. Kim, J. N. Israelachvili, and C. R. Safinya, Proc. Nat. Acad. Sci., 2004, 101,17340-17344.

5 M. Blanc, C.Kléman, Eur. Phys. J. B, 1999, 10, 53-60.

6 L. Z. Ruan, J. R. Sambles, and I. W. Stewart, Phys. Rev. Lett., 2003, 91, 033901.

7 J.-M. Michel, E. Lacaze, M. Alba, M. de Boissieu, M. Gailhanou, and M Goldmann. Phys. Rev. E, 2004, 70, 011709

8 M. Kléman. Points, lines, and walls, J. Wiley, New York, 1983.

9 W.W. Schneider and M.B.Webb. J. Phys. (France), 1984, 45, 273-281

10 P. Boltenhagen, O. Lavrentovich, and M. Kléman. J. Phys. II (France), 1991, 1, 1233-1252

11 Z.Li and O.D.. Lavrentovich, Phys. Rev. Lett., 1994, 73, 280283

12 B. Zappone and E. Lacaze. Phys. Rev. E, 2008, 78, 061704

13 E. Lacaze, J.-P. Michel, M. Alba, and M. Goldman, Phys Rev. $E, 2007,76,041702$

14 J.-P. Michel, E. Lacaze, M. Goldmann, M. Gailhanou, M. de Boissieu, and M. Alba, Phys. Rev. Lett. 2006, 96, 027803

15 E. Lacaze, J.-P. Michel, M. Goldmann, M. Gailhanou, M.de Boissieu, and M. Alba, Physical Review E, 2004, 69, 041705.
16 D. Coursault, B. Zappone, A. Coati, A. Boulaoued, L. Pelliser, D. Limagne, N. Boudet, B.Haj Ibrahim, A. de Martino, M. Alba, M. Goldmann, Y. Garreau, B. Gallas, and E. Lacaze, Soft Matter, 2016, 12, 678-688

17 D. Coursault, B. Haj Ibrahim, L. Pelliser, B. Zappone, A. de Martino, E. Lacaze, and B. Gallas. Optics Express, 2014, 22, 23182-23191

18 I.R. Nemitz, A. J. Ferris, E. Lacaze, and C. Rosenblatt, Soft Matter, 2016, 12, 6662-6668

19 I. Gryn, E. Lacaze, R. Bartolino, and B. Zappone, Adv. Funct. Mater., 2015, 25, 142-149

20 I. Gryn, E. Lacaze, L. Carbone, M. Giocondo, and B. Zappone, Adv. Funct. Mater., 2016, 26, 7122

21 L. Pelliser, et al, Adv. Funct. Mater. 2015, 25, 1719-1726

22 B. Rozic, ACS Nano 2017, 11, 6728-6738]

23 D. Coursault, J. Grand, B. Zappone, H. Ayeb, G. Lévi, N. Félidj, and E. Lacaze, Adv. Mater. 2012, 24, 1461-1465

24. D. Coursault, et al, ACS Nano 2015, 9, 11678-11689

25 D. Johnson and A. Saupe, Phys. Rev. A, 1977, 15, 2079-2085

26 K. Harth and R. Stannarius, Eur. Phys. J. E, 2009, 28, 265-272

27 J.C. Loudet, P. V. Dolganov, P. Patricío, H. Saadaoui, and P. Cluzeau, Phys. Rev. Lett. 2011, 106, 117802

28 R. B. Meyer and P. S. Pershan. Solid State Comm., 1973, 13, 989-992

29 T. J. Scheffer, H. Gruler, and G. Meier, Solid State Comm., 1972, 11, 253

30 J. Fournier and P. Galatola, J. Phys. II (France), 1995, 5, 12971320 ,

31 K. S. Novoselov, A. K. Geim, S. V. Morozov, D. Jiang, Y. Zhang, S.V. Durbonos, I. V. Grigorieva, and A.A. Firsov, Science, 2004, 306, 666-669

32 M. Kléman and O.D. Lavrentovich, Soft Matter Physics, Springer, New York, 2003

33 I. Gryn, L. Pelliser, B. Zappone, M. Goldman, M. Alba, and E. Lacaze, to be published

34 R. Wang, I.M. Syed, G. Carbone, R.G. Petschek, and C. Rosenblatt, Phys. Rev. Lett., 2006, 97, 167802

35 Orsay Group on Liquid Crystals, Solid State Commun., 1971, 9, 653

36 A. Saupe, Mol. Cryst. Liq. Cryst., 1969 7, 59

37 N.D. Mermin, Rev. Mod. Phys., 1979 51, 591

38 E. Lacaze, A. Apicella, M. P. De Santo, D. Coursault, M. Alba, M. Goldmann, and R. Barberi, Soft Matter, 2011, 7, 1078

39 S. Ferjani, Y. Choi, J.Pendery, R. G. Petschek, and C. Rosenblatt, Phys. Rev. Lett., 2010, 104. 257801

$40 \mathrm{~J}$. Pendery, S. Ferjani, C. Rosenblatt, and R. G. Petschek. Europhys. Lett., 2011, 96, 26001

41 Y. Choi, T. Atherton, S. Ferjani, R.G. Petschek, and C. Rosenblatt, Phys. Rev. E, 2009, 80, 060701

42 R. Basu, C. Rosenblatt, and R. P. Lemieux, Liq. Cryst., 2012 39, 199 ,

43 R. Basu, D. Kinnamon, and A. Garvey, J. Appl. Phys., 2015 118, 114302 